\title{
MULTISCALE ISOGEOMETRIC OPTIMIZATION FOR CELLULAR STRUCTURE WITH MULTIPLE PROTOTYPES
}

\author{
Chen Yu ${ }^{1, *}$, Qifu Wang ${ }^{1}$, Chao Mei ${ }^{1}$ and Zhaohui Xia ${ }^{1}$ \\ ${ }^{1}$ National Enterprise Information Software Engineering Research Center, School of Mechanical \\ Science and Engineering, Huazhong University of Science and Technology \\ Wuhan 40074, China \\ hustcadyuchen@hust.edu.cn, https://www.researchgate.net/profile/Chen_Yu90
}

Key words: Isogeometric Analysis, Topology Optimization, Cellular Structure, Multiple Prototypes, Transformation Method.

\begin{abstract}
This paper presents a multiscale concurrent isogeometric design method for the cellular structure composed of the multiple prototype microstructures. Based on the unified-skeleton modeling scheme, the graded microstructures obtained from a same prototype have similar topology and good connectivity between each other. For the different prototypes, the configurations are rearranged and added the thin-wall material in common interfaces. Thus, the geometric features as well as the structural skeletons can have a smooth transition. Through combining the numerical homogenization method with the interpolation technique, the effective properties of graded microstructures can be estimated efficiently. For the multiscale scheme, the allocation of microstructures is optimized by a material distribution-based method while the configurations of prototypes are designed by the isogeometric parameterized level set method. The proposed method inherits the high accuracy and high efficiency of isogeometric analysis and the smooth boundaries and distinct interfaces of level set method. The cellular structure can be freely customized with favorable connectivity.
\end{abstract}

\section{INTRODUCTION}

The artificial functionally graded materials consisting of the spatially-varying cellular microstructures have been extensively studied and utilized in engineering applications [1]. Instead of choosing different base material, the key issue for gaining the desired performance is to design configurations and layouts of cellular materials. Currently, the so-called concurrent design or hierarchical design [2] are the effective methods for multiscale cellular structure. By optimizing the prototype microstructure and deriving the corresponding graded microstructure, the assembled macrostructure shows flexible and modular design freedom. Moreover, the multiple prototypes [3] are employed to extend the diversity of microstructure, thereby realizing the complex and spatially varying cellular structure. However, it is difficult to maintain the connectivity between adjacent microstructures without extra geometric constraints in design process. Hence, this paper proposes a multiscale concurrent design method for cellular structure with multiple well-connected prototypes.

The multiscale design for cellular structure can be divided into the single-scale structure design in both macro scale and micro scale. The topology optimization is an effective structure optimization tool for achieving the optimal material distribution under predefined constraints. Compared to the density-based 
representation [4] such as the homogenization method, solid isotropic material with penalization method (SIMP) and evolutionary structural optimization method (ESO), the boundary-based representation [5] avoid the numerical artifacts like checkerboard and mesh dependency without the extra filtering process and stabilization. The level set method (LSM) can flexibly handle the complex topology evolution with distinct interfaces. By adopting the non-uniform rational basis splines (NURBS) as basis functions for both parameterized level set model and isogeometric calculation model [6], the configuration design for prototypes inherits the high accuracy and high efficiency [7].

For bridging the design between different scales, the asymptotic expansion theory based numerical homogenization method [8] is employed to estimate the effective elasticity tensors for microstructures. Thus, the configuration design of microstructure can be regarded as the inverse design problem for achieving desired properties. Da et al. [9] optimized the material microstructures for maximum effective elastic modulus. Ye et al. [10] utilized the inverse method to design the gradually stiffer mechanical meta-material for cushioning and vibration damping in engineering. For spatially varying graded microstructures, the effective property is no longer fixed and can be used to analyze the displacement field of macro structure providing that the neighboring cellular microstructures have sufficiently small change in gradient of shape and property. Combining the shape metamorphosis method [11] with unified-skeleton modeling scheme [12], the interpolation process of effective property estimation can be simplified by fitting function.

However, the scale separate assumption in homogenization method gives rise to the uncertainty for connectivity of microstructural geometry. The filtering technique [13] and predefined connected region [14]

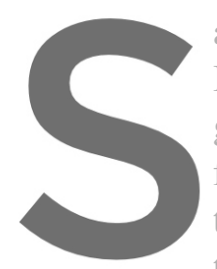
as the candidates of ge In addition, the transition generate the transition in from the same prototyp the connected component the cellular structure compesed of the ming
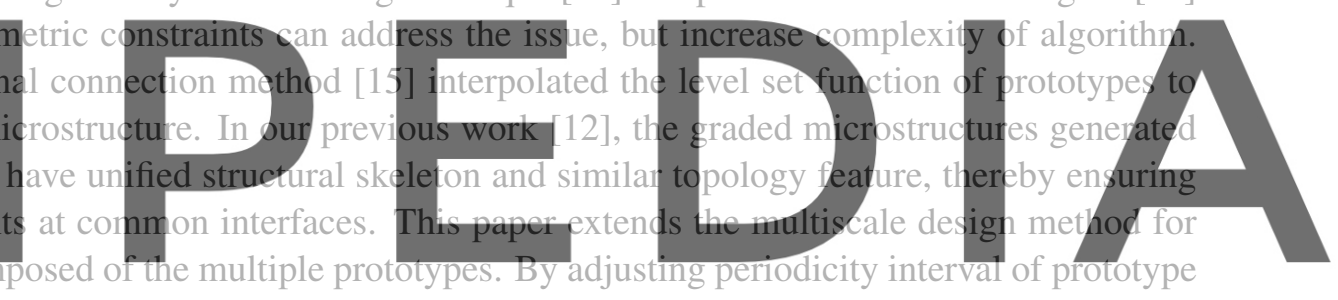
microstructure, the different structural skeletons as well as components connect together as a whole. In

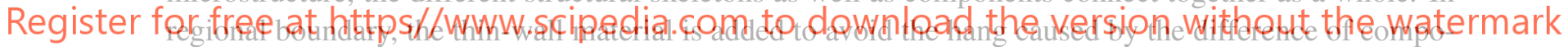
nent numbers.

In this paper, we achieve a multiscale concurrent isogeometric topology optimization for the cellular structure derived from the multiple prototypes. In Section 2, by integrating level set method and isogeometric analysis [16], the proposed method inherits the high accuracy and high efficiency. Then the unified-skeleton modeling scheme and the periodicity interval adjusting technology are introduced to construct the cellular structure with well connectivity. Section 3 presents the formulation and the sensitivity analysis. The numerical examples in Section 4 demonstrate the validity.

\section{MODELING FOR CELLULAR STRUCTURE}

\subsection{Isogeometric level set method}

In IGA, the NURBS [17] is utilized for the numerical discretization. For the B-spline with $p$ order and $n$ spline basis function, the non-decreasing knot vector $\mathrm{H}=\left\{\vartheta_{1}, \vartheta_{1}, \cdots, \vartheta_{n+p+1}\right\}$ describes the parametric coordinates of a curve. Then the B-spline functions are recursively defined according to the Cox-de Boor 
formula [18] as:

$$
\left\{\begin{array}{l}
B_{i, 0}(\vartheta)= \begin{cases}1 & \text { if } \vartheta_{i} \leq \vartheta<\vartheta_{i+1} \\
0 & \text { otherwise }\end{cases} \\
B_{i, p}(\vartheta)=\frac{\vartheta-\vartheta_{i}}{\vartheta_{i+p}-\vartheta_{i}} B_{i, p-1}(\vartheta)+\frac{\vartheta_{i+p+1}-\vartheta}{\vartheta_{i+p+1}-\vartheta_{i+1}} B_{i+1, p-1}(\vartheta)
\end{array}\right.
$$

They constitute a partition of unity. When $k$ is the multiplicity of the knots, the continuity among knot spans is $C^{p-k}$. Thus, the curve shows favorable smoothness. The two-dimensional B-spline basis function is constructed as $B_{i, p}^{j, q}(\vartheta, \zeta)=B_{i, p}(\vartheta) B_{j, q}(\zeta)$, which can be further used to generate the bivariate B-spline surface. NURBS basis functions as extension of B-spline are constructed by introducing the positive weight $\omega_{i}$, then the two-dimensional NURBS basis functions are expressed as:

$$
N_{i, p}^{j, q}(\vartheta, \varsigma)=\frac{B_{i, p}(\vartheta) B_{j, q}(\varsigma) \omega_{i, j}}{\sum_{k=1}^{n} \sum_{l=1}^{m} B_{k, p}(\vartheta) B_{l, q}(\varsigma) \omega_{k, l}}
$$

Based on the basis functions, a NURBS surface can be defined as a bivariate piece-wise rational function as:

$$
R(\vartheta, \varsigma)=\sum_{i=1}^{n} \sum_{j=1}^{m} N_{i, p}^{j, q}(\vartheta, \varsigma) P_{i, j}
$$
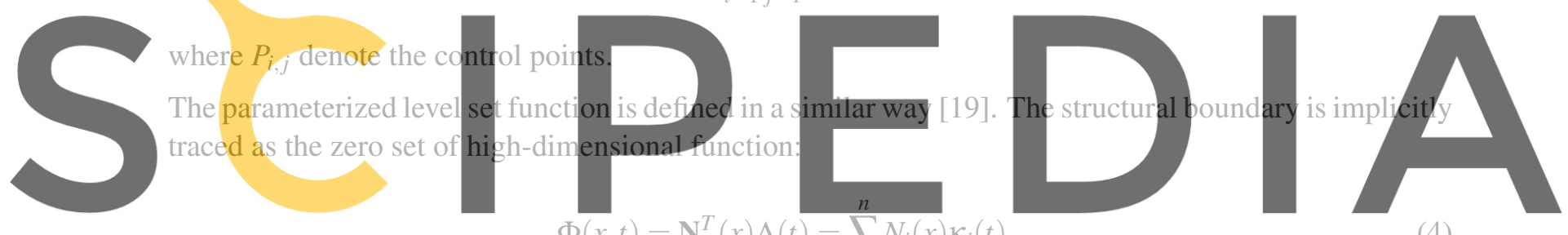

$\Phi(x, t)=\mathbb{N}^{T}(x) \Lambda(t)=\sum N_{i}(x) \kappa_{i}(t)$

(4)

Register for free at https//www.scipedia.com to download the version without the watermark

Difference both side of Eq. (4) with respect to pseudo time $t$, the Hamilton-Jacobi equation is obtained as:

$$
\mathbf{N}^{T}(x) \frac{\partial \Lambda(t)}{\partial t}-v_{n}\left|(\nabla \mathbf{N}(x))^{T} \Lambda(t)\right|=0
$$

Then the normal velocity can be represented as $v_{n}=\left(\mathbf{N}^{T}(x) /\left|(\nabla \mathbf{N}(x))^{T} \Lambda(t)\right|\right)(\partial \Lambda(t) / \partial t)$.

In order to calculate the initial level set values as well as the expansion coefficients, the Greville abscissae is employed as the selection strategy of collocation points. When order and control point number of NURBS are $p, m$ respectively. The Greville abscissae are defined by:

$$
\zeta_{i}=\frac{1}{p}\left(\xi_{i+1}+\xi_{i+2}+, \cdots,+\xi_{i+p}\right), \quad i=1,2, \cdots, m
$$




\subsection{Geometry model of cellular structure with multiple prototypes}

Referring to [12], the level set based prototype microstructure is used to generate the unified-skeleton graded microstructures to ensure the connectivity between adjacent cells. In [20], the structural skeletons are defined as the center set of the maximal inscribable sphere. As shown in Figure 1, the blue dotted line represents the skeleton, at point $x_{s}$ of which the normal and tangential direction are defined as $n\left(x_{s}\right), \tau\left(x_{s}\right)$. Due to the signed-distance modeling scheme, the level set function meets the equation:

$$
\frac{\partial \Phi\left(x_{s}\right)}{\partial n\left(x_{s}\right)}=0, \quad \frac{\partial \Phi\left(x_{s}+n \cdot d\right)}{\partial n\left(x_{s}+n \cdot d\right)}<0
$$

Setting the level set values at structural skeleton as maximum of $\Phi$, reconstructing the level set function as

$$
\widetilde{\Phi}(x, t)= \begin{cases}\frac{R_{b}}{R_{s}+R_{b}} \max (\Phi(x, t)) & x \in \Omega \\ \frac{R_{b}}{R_{s}-R_{b}} \max (\Phi(x, t)) & x \notin \Omega\end{cases}
$$

where $R_{b}$ and $R_{s}$ denote the minimum distances of point $x$ to structural boundary and skeleton respectively,
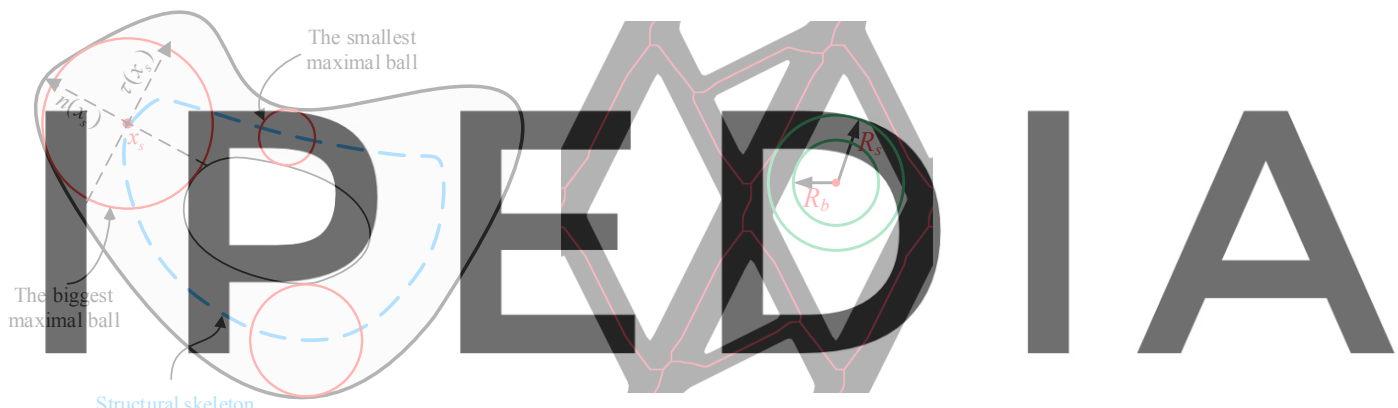

Register for free at https//www.scipedia.com to download the version without the watermark Figure 1: Based on structural skeleton, reconstructing the level set function.

According to [11], for reproducing the property of the base material, the solid microstructure must be contained in the graded microstructures. Therefore, the geometric description of microstructures should retain the feature of prototype. The level set function of solid microstructure and void microstructure are defined as:

$$
\left\{\begin{array}{l}
\tilde{\Phi}_{\text {solid }}(x)=\tilde{\Phi}(x)-\min (\tilde{\Phi}) \\
\tilde{\Phi}_{\text {void }}(x)=\tilde{\Phi}(x)-\max (\tilde{\Phi})
\end{array}\right.
$$

Then the graded cellular microstructures are expressed by:

$$
\tilde{\Phi}_{g m}(x)=(1-f(\rho)) \tilde{\Phi}_{\text {void }}(x)+f(\rho) \tilde{\Phi}_{\text {solid }}(x)
$$

where $\rho$ denotes the relative density. $f(\rho)$ is the increasing function which can be solved by the method of bisection. 
For the cellular structure with multiple prototypes, we rearranged and added the thin-wall material in common interfaces. As shown in Figure 2, several prototypes from the reference [6] are arranged from left to right. Because they have different topology as well as the structural skeletons, there are some mismatch and hang in the common interfaces. In consideration of the periodicity of prototypes, the microstructure can be freely captured providing fixed length of interval. Setting the points where level set function reaches the maximum value as new nodes $x_{\text {new }}$, the skeleton will go through them. Then all the skeletons of different prototypes connect to each other at the nodes.

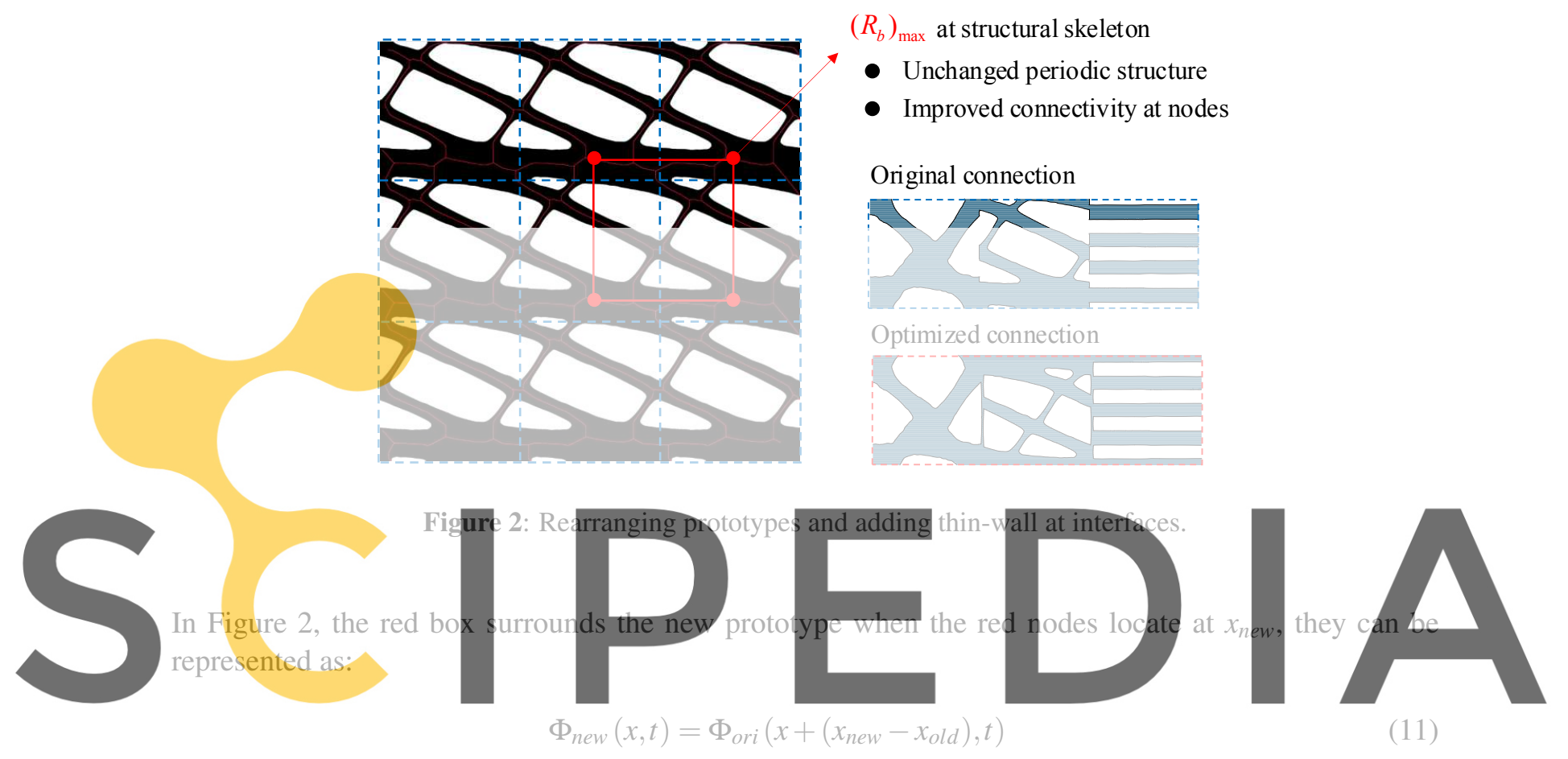

Register for free at https//www.scipedia.com to download the version without the watermark Although the rearranged prototype microstructure is different from the original one, the periodically assembled macrostructures have roughly the same topology. The nodes having the maximal level set value means that the microstructural components for connection possess the biggest size. Hence, this method can improve the connectivity at nodes effectively.

However, in consideration of difference of component number, there may be the mismatches of geometric features at middle part of interface. The thin-wall material is added to address this issue.

\subsection{Estimation of effective property}

Homogenization method is used to calculate the effective properties of microstructures. Via the asymptotic homogenization, a physical field such as the displacement field can be expressed as an asymptotic expansion:

$$
u^{v}(x)=u_{0}(x, y)+v u_{1}(x, y)+v^{2} u_{2}(x, y)+\cdots
$$


where the variables at macroscopic and microscopic are represented as $x, y$. The aspect ratio $v=x / y$ is very small. Only considering the first order of Eq. (12), the effective stiffness tensor is calculated as:

$$
\mathbf{D}_{p q r s}=\frac{1}{|V|} \int_{V_{s}} M_{p q k l} \mathbf{D}_{k l m n} M_{r s m n} d V
$$

$V$ is the solid part of the cell, and $|V|$ denotes its volume. $\mathbf{D}_{k l m n}$ is the locally elastic matrix, vary from the position of $d V$. Driving from the test strain $\varepsilon_{k l}^{0}, \varepsilon_{m n}^{0}$, the displacement field $u^{p q}, u^{r s}$ as well as the strain field $\varepsilon_{k l}^{*}\left(u^{p q}\right), \varepsilon_{m n}^{*}\left(u^{r s}\right)$ can be yielded. Then, the local structural tensor $M_{p q k l}$ and $M_{r s m n}$ can be calculated as:

$$
\left\{\begin{array}{l}
M_{p q k l}=\varepsilon_{k l}^{0}-\varepsilon_{k l}^{*}\left(u^{p q}\right) \\
M_{r s m n}=\varepsilon_{m n}^{0}-\varepsilon_{m n}^{*}\left(u^{r s}\right)
\end{array}\right.
$$

With regard to the similar shape and property of graded microstructures, the regularized effective elasticity tensor is introduced. Ignoring the parameters of base material, the effective modulus and elasticity matrix are represented as:

$$
\left\{\begin{array}{l}
E^{H}=(1+\mu)(1-\mu) E_{\text {pqrs }} / E_{\text {base }} \\
D^{H}=(1+\mu)(1-2 \mu) D_{\text {pqrs }} /\left[(1-\mu) D_{\text {base }}\right]
\end{array}\right.
$$

Through the interpolation between relative density $\rho_{i}$ and $\rho_{i+1}$, the effective property of the arbitrary

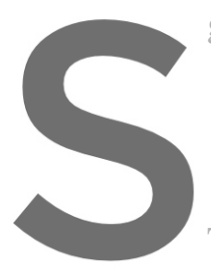
graded cell can be expressed as:

Thus, for the isogeometric paranneterized level set
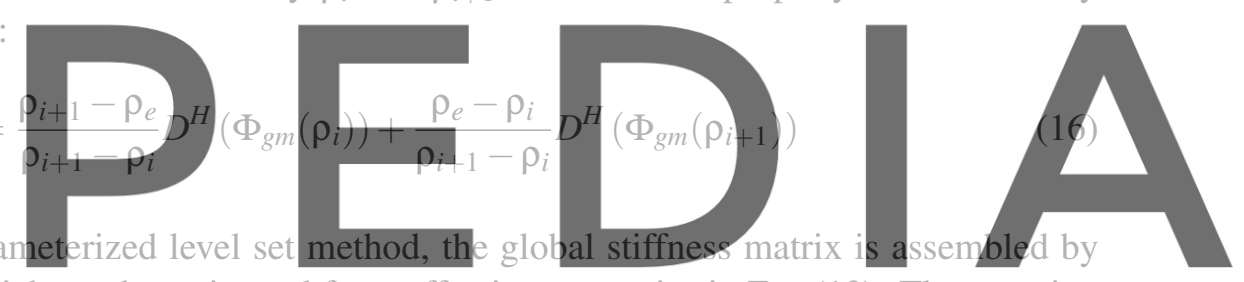

the element stiffness matrix which can be estimated from effective properties in Eq. (13). The equation

\section{Register for describegt asittps//www.scipedia.com to download the version without the watermark}

$$
K=\sum_{e=1}^{N} k_{e} \Pi=\sum_{e=1}^{N} \int_{V} \frac{\mathbb{B}_{e}^{T}\left[(1-\mu) D_{\text {base }}\right] \mathbb{D}_{e}^{H} \mathbb{B}_{e}}{(1+\mu)(1-2 \mu)}\left|J_{1}\right||| J_{2} \mid d V \Pi
$$

where $\Pi$ denotes the selection matrix in the common notation. Jacobian $\mathbf{J}_{1}$ and $\mathbf{J}_{2}$ represent the transformation relationship among the integration parametric space, NURBS parametric space and the physical space.

\section{FORMULATION AND SENSITIVITY}

The proposed multiscale design is divided into macro scale and micro scale. On one hand, the configurations of multiple prototypes are designed, the corresponding graded microstructures are evolved synchronously. On the other hand, the prototypes are adjusted for their periodicity interval and added the thin-wall materials at interfaces. The distributions of the corresponding microstructures are optimized. 
The formulations of topology optimization are written as:

$$
\begin{array}{lll}
\text { Design variables : } & \mathrm{T}=\left(\rho_{1}, \rho_{2}, \cdots, \rho_{N}\right), \quad \Lambda^{Z}=\left(\kappa_{1}^{Z}, \kappa_{2}^{Z}, \cdots, \kappa_{n}^{Z}\right) Z=1,2, \cdots, P \\
\text { Objective }: & & J=\mathbf{F}^{T} \mathbf{U}=\mathbf{U}^{T} \mathbf{K} \mathbf{U}=\sum_{e=1}^{N} \mathbf{U}_{N_{e}}^{T}\left(\int_{\Omega} \mathbf{B}_{e}^{T} \mathbf{D}^{H}\left(\chi_{e}, \Phi^{Z}\left(\Lambda^{Z}, \rho_{e}\right)\right) \mathbf{B}_{e} d \Omega\right) \mathbf{U}_{e} \\
\text { Constraints : } & \mathbf{F}=\left\{\sum_{e=1}^{N} \int_{V} \frac{\mathbf{B}_{e}^{T}\left[(1-\mu) D_{\text {base }} \mathbf{D}_{e}^{H} \mathbf{B}_{e}\right.}{(1+\mu)(1-2 \mu)}\left|\mathbf{J}_{1}\right|\left|\mathbf{J}_{2}\right| d V \Pi\right\} \mathbf{U} \\
& G=\sum_{e=1}^{N} \rho_{e} V_{0}-V_{\max } \leq 0 \\
& g_{Z}=V_{Z}-V_{Z, \max }=\int_{Y_{Z}} H\left(\Phi_{Z}\right) d Y_{Z}-V_{Z, \max } \leq 0 \\
& 0<\rho_{\min } \leq \rho_{e} \leq 1 \alpha_{\min } \leq \alpha^{Z} \leq \alpha_{\max }
\end{array}
$$

where $\Lambda^{Z}$ are the expansion coefficients set of the multiple prototypes. The relative densities of microstructures are macro-scale design variables to decide the location of the graded ones. The objective function is influenced by a lot of parameters. Thus, it is necessary to find out a simplified representation for implementing the optimization.

The problem will be solved by the gradient based algorithms. Then the method of moving asymptotes method is employed to evolve the design variables in both scales. For the sensitivity analysis, the derivatives of objective function to the densities are expressed as:

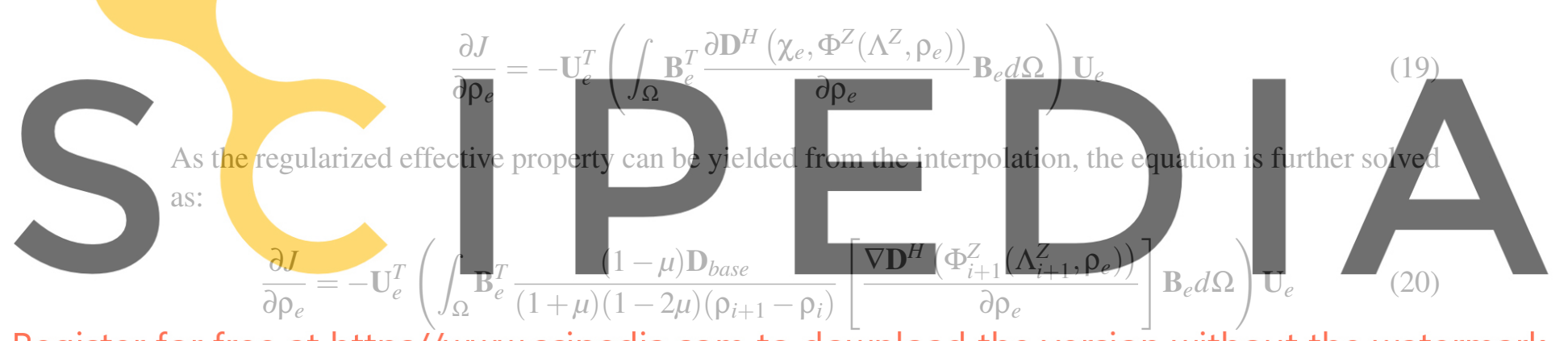

Register for free at https//www.scipedia.com to download the version without the watermark

Although the prototype is updated in process of optimization, the modeling scheme for the graded mi-

crostructures guarantees that the variation trend of macroscopic response with respect to the relative density is not tremendous. Hence, the evolution of macro-scale design is stable and efficient.

In micro scale, the number of the design variables is $n \times P$, which can be decoupled to perform the sensitivity analysis. It can be seen from Eq. (18) that the regularized effective properties of graded microstructures are too complex to describe in an analytical form. Without special numerical equation treatment, there will be huge amount of computation required in the analysis.

Taking the prototype microstructure as a representation, all the graded ones are related to it. Thus, the sensitivity can be described as:

$$
\frac{\partial J}{\partial \kappa_{e}^{Z}}=-\sum_{e=1}^{M_{Z}} \mathbf{u}_{e}^{T}\left(\int_{V_{e}} \mathbf{B}_{e}^{T} \frac{\partial \mathbf{D}^{H}\left(\chi_{e}, \Phi^{Z}\left(\Lambda^{Z}, \rho_{e}\right)\right)}{\partial \kappa_{e}^{Z}} \mathbf{B}_{e} d V_{e}\right) \mathbf{u}_{e}
$$

where $M_{Z}$ is number of graded microstructures from prototype $\mathrm{Z}$, the equations are parallel in a step of optimization. Referring to the shape derivative [21] and the chain rules, the derivatives of objective 
function are extended as:

$\frac{\partial J}{\partial \kappa_{e}^{Z}}=-\sum_{e=1}^{M_{Z}} \mathbf{u}_{e}^{T}\left(\int_{V_{e}} \mathbf{B}_{e}^{T} \frac{1}{\left|V_{N e}\right|} \int_{V}\left(\varepsilon_{k l}^{0}-\varepsilon_{k l}^{*}\left(u^{p q}\right)\right) \mathbf{D}\left(\varepsilon_{m n}^{0}-\varepsilon_{m n}^{*}\left(u^{r s}\right)\right) H(\Phi) \delta(\Phi) N(x) d V \mathbf{B}_{e} d V_{e}\right) \mathbf{u}_{e}(22)$

$N(x)$ is the NURBS basis function which is linked to the collocation point and expansion coefficient. The variables of integration are shared for the estimation of effective property and calculation of derivative. Therefore, it prefers to adopt the parallel computation which runs on Graphics Processing Unit using Computing Unified Device Architecture.

\section{NUMERICAL EXAMPLES}

The multiscale minima compliance problems are tested in this Section. We assume that the Young's modulus and Poisson's radio of base material are 1 and 0.3 respectively. For the alternative one-scale evolution, the optimization will terminate until the relative differences of objective function and design variables are below 0.01 .

In Figure 3(a), the cantilever beam is designed for the cellular structure with multiple prototypes. The beam with $60 \times 20$ cells is fixed at the left edge, and the vertical force is applied at center of right. There are five prototypes for generating the corresponding microstructures. The distributions of them are optimized at the predefined subdomain which are divided from calculating the orientations of major principal stresses.

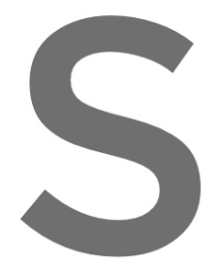

With respect to the uncor mented. By adjusting the structure is almost uncha terial must be allocated a solution is illustrated, and
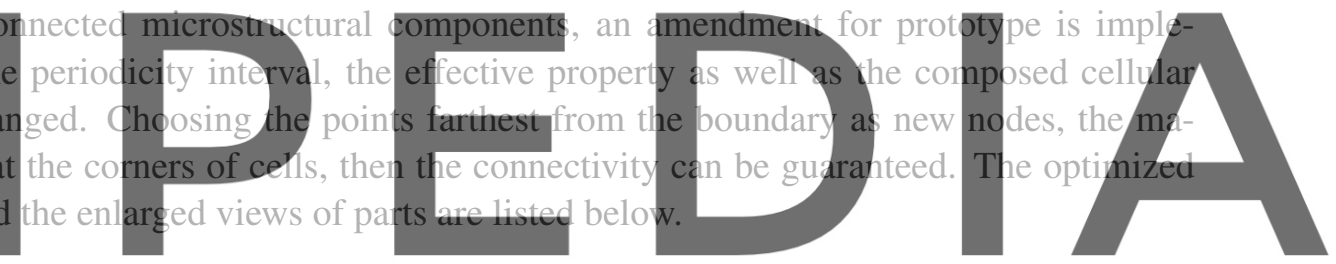

Register for free aththps//www.scipedia.com to dowhload the version without the watermark
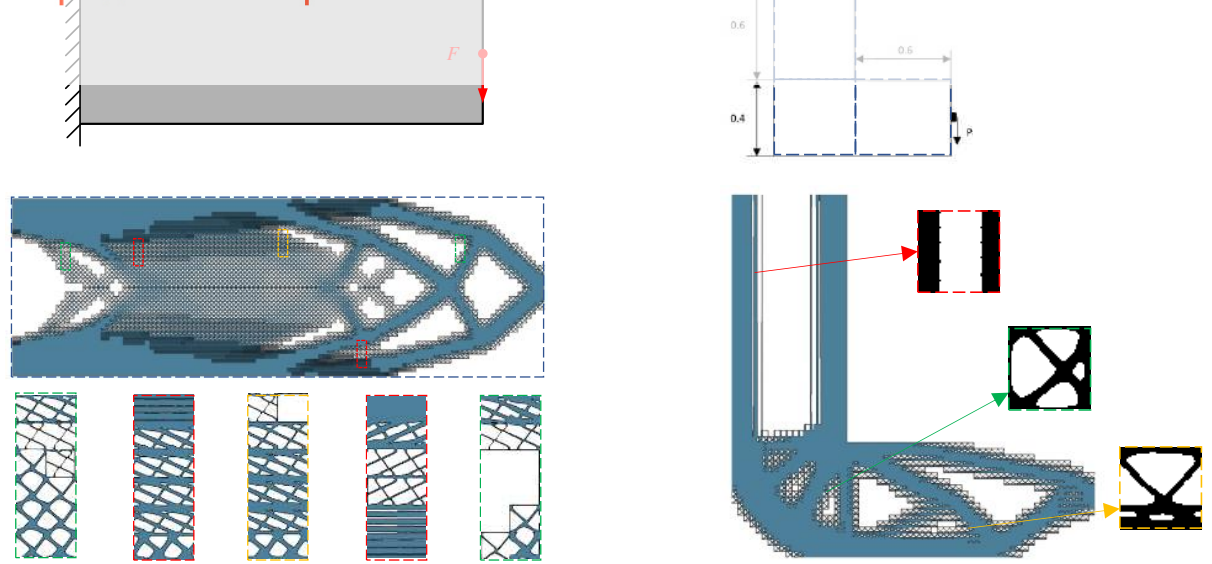

Figure 3: Numerical examples of cantilever beam and L-shape structure. 
Another example is the L-shape structure shown in Figure 3(b). The structure is fixed at the upper left side, and a vertical force is applied at the lower right. The macrostructure is divided into three groups which correspond to individual prototype and derived graded microstructures. In comparison to one type of cells, the employment of the multiple prototypes brings more design freedom. The configurations of prototypes can be customized according to the local stress condition. The processing of boundary connections promoted the optimized solution closing to engineering practice.

In addition, the effect of more groups for configurations and distributions of prototypes is studied. The optimized results show the similar distribution to the SIMP method in macro scale. The micro-scale topology shows more detail features as the groups increase.

\section{CONCLUSIONS}

This paper proposes a multiscale concurrent isogeometric design method for cellular structure constituted by multiple prototype microstructures. There are several highlights as:

- Our work integrates the isogeometric analysis, thus the method has a higher accuracy and fewer design variables.

The structural skeleton is introduced to generate the graded microstructures. Then the microstructures have unified skeleton and similar topology, thereby promoting the connectivity of the components.

For the different prototypes, the periodicity intervals are rearranged and the thin-wall material is

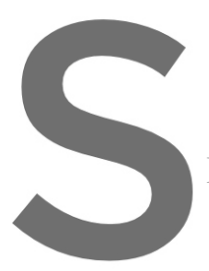
added at the common interfaces. Thus, the geometric features as well as the structural skeletors can have a smooth transition

REFERENCES

[1] Li, H., Gao, L., Li, H. and
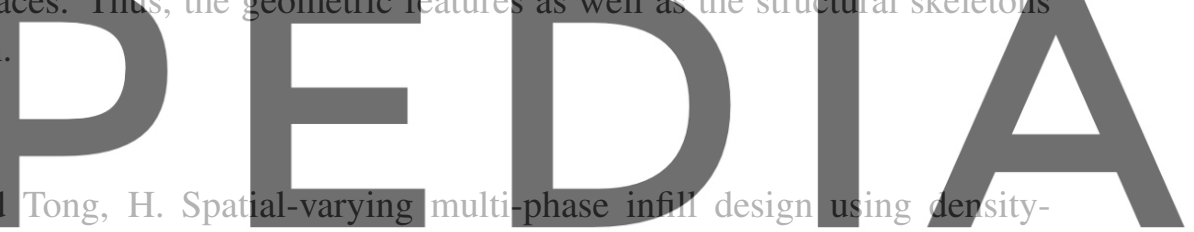

based topology optimization. Computer Methods in Applied Mechanics and Engineering (2020)

Register for free at 37 teps//www.scipedia.com to download the version without the watermark

[2] Long, K., Han, D. and Gu, X. Concurrent topology optimization of composite macrostructure and microstructure constructed by constituent phases of distinct Poisson's ratios for maximum frequency. Computational Materials Science (2017) 129:194-201.

[3] Wu, Z., Xia, L., Wang, S. and Shi, T. Topology optimization of hierarchical lattice structures with substructuring. Computer Methods in Applied Mechanics and Engineering (2019) 345:602-617.

[4] Bendsøe, M.P. and Sigmund, O. Material interpolation schemes in topology optimization. Archive of Applied Mechanics (1999) 69:635-654.

[5] van Dijk, N.P., Maute, K., Langelaar, M. and van Keulen, F. Level-set methods for structural topology optimization: A review. Structural and Multidisciplinary Optimization (2013) 48:437-472.

[6] Yu, C., Wang, Q., Mei, C. and Xia, Z. Multiscale isogeometric topology optimization with unified structural skeleton. Computer Modeling in Engineering and Sciences (2020) 122:779-803.

[7] Wang, Y., Liao, Z., Shi, S., Wang, Z. and Poh, L.H. Data-Driven Structural Design Optimization for Petal-Shaped Auxetics Using Isogeometric Analysis. Computer Modeling in Engineering and 
Sciences (2020) 122:433-458.

[8] Sigmund, O. Materials with prescribed constitutive parameters: An inverse homogenization problem. International Journal of Solids and Structures (1994) 31:2313-2329.

[9] Da, D., Cui, X., Long, K., Huang, G. and Li, G. Design of material microstructures for maximum effective elastic modulus and macrostructures. Engineering Computations (2018) 35:622-640.

[10] Ye, M., Gao, L. and Li, H. A design framework for gradually stiffer mechanical metamaterial induced by negative Poisson's ratio property. Materials \& Design (2020) 192:108751.

[11] Wang, Y., Chen, F. and Wang, Y. Concurrent design with connectable graded microstructures. Computer Methods in Applied Mechanics and Engineering (2017) 317:84-101.

[12] Yu, C., Wang, Q., Mei, C. and Xia, Z. An efficient multiscale concurrent design method using fitting function. International Journal of Materials and Product Technology (2020) 61:202-227.

[13] Radman, A., Huang, X. and Xie, Y. Topology optimization of functionally graded cellular materials. Journal of Materials Science (2013) 48:1503-1510.

[14] Li, H. Luo, Z., Gao, L. and Qin, Q. Topology optimization for concurrent design of structures with multi-patch microstructures by level sets. Computer Methods in Applied Mechanics and Engineering (2018) 331:536-561.

[15] Liang, S., Gao, Li., Zheng, Y. and Li, H. A Transitional Connection Method for the Design of Functionally Graded Cellular Materials. Applied Sciences (2020) 10:7449.

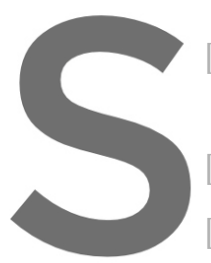

[16] Wang, Y. and Benso optimization. Computationd

[17] Piegl, L. and Tille,
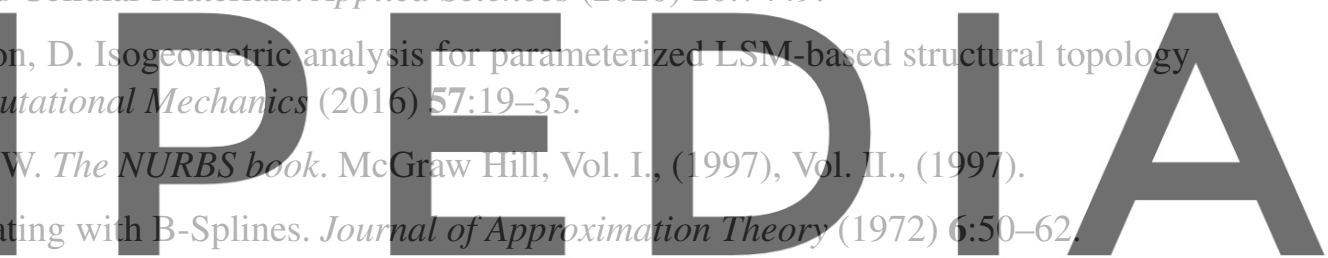

[19] Wei, P., Yang, Y., Chen, S. and Wang, Y. A Study on Basis Functions of the Parameterized Level

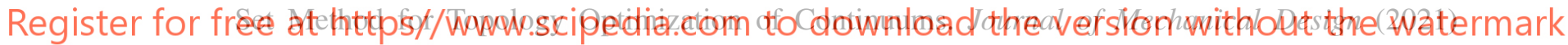
143:041701.

[20] Gao, J., Song, B. and Mao, Z. A novel approach for length scale control in structural topology optimization. Engineering Optimization (2019) 51:1668-1686.

[21] Wang, Y., Liao, Z., Ye, M., Zhang, Y., Li, W. and Xia, Z. An efficient isogeometric topology optimization using multilevel mesh, MGCG and local-update strategy. Advances in Engineering Software (2020) 139:102733. 